\title{
SciencePG-Discovery-Time series analysis of global/Korean earthquake occurrence and recent GPS crustal displacement recorded at Daejeon, Korea
}

\author{
Sung-Ho Na ${ }^{1}$, Dork Sahagian ${ }^{2,}$, , Pil-Ho Park ${ }^{1}$, Tae-Woong Chung ${ }^{3}$, Tae-Seob Kang ${ }^{4}$ \\ ${ }^{1}$ Korea Astronomy and Space Science Institute, Daejeon, Republic of Korea \\ ${ }^{2}$ Earth and Environmental Sciences, Lehigh University, Bethlehem, PA, USA \\ ${ }^{3}$ Sejong University, Seoul, Republic of Korea \\ ${ }^{4}$ Pukyung National University, Pusan, Republic of Korea
}

Email address:

sunghona@kasi.re.kr(S. Na), dos204@lehigh.edu(D. Sahagian)

\section{To cite this article:}

Sung-Ho Na, Dork Sahagian, Pil-Ho Park, Tae-Woong Chung, Tae-Seob Kang. SciencePG-Discovery - Time Series Analysis of Global/Korean Earthquake Occurrence and Recent GPS Crustal Displacement Recorded at Daejeon, Korea. Science Discovery.

Vol. 1, No. 2, 2013, pp. 6-15. doi: 10.11648/j.sd.20130102.11

\begin{abstract}
Inherent periodicities are observed in earthquake occurrence for three kinds of time series - one is based on a global earthquake occurrence record for the last two hundred years, while the others are based on either historical or recent Korean earthquake records. Above numerous minor peaks in the global earthquake occurrence spectra, there exist a few peaks including Chandler periodicity over 95 percent significance level. The global earthquake triggering mechanisms at each identified period are not entirely clear. An outstanding one-year periodicity has been found for the historical Korean earthquakes. This one-year periodicity is interpreted to reflect seasonal forcing that results in annual oscillation of Korean crust, as recorded over twelve years of GPS monitoring at Daejeon (DAEJ), one of International GNSS Service (IGS) permanent stations.
\end{abstract}

Keywords: Earthquake, Spectral Analysis, Chandler Wobble, Seasonal Oscillation, GPS

\section{Introduction}

Large and small earthquakes occur over the world without warnings. The well known 'frequency-magnitude relation of earthquake occurrence,' is written as $\log _{10} N=a-b M$, where $N$ is the number of earthquakes of magnitude greater than $M[1]$. Two constants $a$ and $b$ vary depending on region and time span. Within certain limits, such as magnitude threshold, this relation generally remains as valid. According to the relation, earthquake occurrence frequency decreases with magnitude increase. The 'frequency' involved with the relation is evidently 'total number of events per a given time', and is not the one associated with 'periodic cycle'. In fact, earthquake occurrences are almost completely unpredictable. A few recent studies suggest that geophysical signals differ before and after large earthquakes[2-4]. Through analysis of satellite gravity data, Han et al. found gravity change of 30 microgal induced by the 2004 Sumatra earthquake[2]. Park et al. found electric potential disturbance of $2.5 \mathrm{mV}$ during the 2004 Parkfield earthquake[3]. Lee et al. reported the ionospheric turbulence originated by the 2004 Sumatra earthquake[4]. Different types of information and knowledge have been accumulated for seismic activity around the globe. Nowadays, many geodetic datasets - mostly GPS derived, reflect co-seismic or post-seismic displacement associated with large earthquakes[5-7]. Bock et al. developed to combine GPS record and integrated-accelerogram to infer reliable estimate of strong motion[5]. Ozawa et al. reported seismic and coseismic slip due to 2011 Tohoku earthquake[6]. Heki described the displacement monitored by GPS due to two large earthquakes; the 2011 Tohoku earthquake and the 2010 Feb 27 Chile earthquake[7]. However, the longstanding objective - 'to predict earthquakes in advance' - is still not realized. Spectral analysis of earthquake occurrence over short time span usually fails to reveal any periodicity above noise level.

Tidal perturbation has been ascribed to trigger earthquakes by careful investigation of large numbers of global earthquakes. Métivier et al. found a correlation between Earth body tide and earthquake occurrence[8]. 
Cochran et al. found that shallow thrust earthquakes occurred in phase with tidal stress three times over background random effects[9]. Tolstoy et al. reported a strong correlation between seamount microearthquakes and ocean tidal loading[10]. Tanaka et al. found that tidally induced shear stress affects earthquake occurrence, particularly for reverse faults[11]. It is noted here that negative assertions about tidal triggering have been published as well (for example, see[12-13]). There may be other geophysical phenomena that serve to trigger earthquakes. For example, episodic slow slip events occurring at several circum-Pacific subduction zones have shown periodicity of 13-16 months, which could be attributed to planetary deformation associated with the Chandler wobble[14].

If a periodic geophysical process consistently contributes to the stress field, then that periodicity might be reflected in earthquake occurrence time series. In this study, spectra for time series of earthquake occurrence were acquired for the following datasets; (i) National Geophysical Data Center (NOAA) global earthquake data for the last two centuries, (ii) local earthquakes reported in Korea during last millennium, and (iii) recent Korean earthquakes recorded by seismometers. The results reveal fringes of certain periodicities, including the Chandler period, in the global earthquake records, and a clear one-year periodicity in the Korean earthquakes. Although their signals are weak, these periodicities manifest the earthquake triggering effects by the two important geophysical phenomena - Chandler wobble and seasonal perturbations in the Earth's crust.

Small crustal oscillation of the Korean peninsula has been sought by analyzing GPS data. Geodetic GPS measurements have been carried at several sites in Korea over the last decade. There are now nine IGS permanent sites operating in Korea, and many other GPS stations are maintained. For this study, data from the longest operating IGS site - DAEJ, are processed to see whether any noticeable periodic movement exists.

\section{Data Analysis}

\subsection{NOAA Global Earthquake Record}

A list of global earthquakes was acquired from the NOAA web sites (National Geophysical Data Center, NOAA). A comparable dataset can also be obtained from USGS. The list includes 3373 earthquakes $(\mathrm{M}>4)$ recorded between March 1800 and December 1994. On average, this occurrence corresponds to about three earthquakes recorded per two months. In Table 1, are given some excerpts from the list for the years 1812 and 1981. They contain information regarding date, location, focal depth, magnitude estimate.
Table 1. List of large global earthquakes in 1812 and 1981 from NOAA website (National Geophysical Data Center). The columns include information regarding date, location, focal depth, and magnitude of each recorded seismic event. Negative latitude/longitude refers to southern/western hemisphere, respectively.

\begin{tabular}{|c|c|c|c|c|c|c|c|}
\hline Year & Mon & Date & Time & Lat & Long & Dep & Mag \\
\hline 1812 & Jan & 23 & 15 & 36.3 & -89.6 & - & 8.4 \\
\hline 1812 & Feb & 7 & $9: 45$ & 36.5 & -89.6 & - & 8.8 \\
\hline 1812 & Mar & 8 & - & 43.7 & 83.0 & - & 8.0 \\
\hline 1812 & Mar & 22 & - & 41.9 & 12.5 & - & - \\
\hline 1812 & Mar & 23 & - & 43.7 & 5.7 & - & - \\
\hline 1812 & Mar & 26 & $20: 07$ & 10.6 & -66.9 & 33 & 7.7 \\
\hline 1812 & Jun & 23 & - & 43.3 & 5.4 & - & - \\
\hline 1812 & Nov & 11 & $10: 50$ & 18 & -76.5 & - & - \\
\hline 1812 & Dec & 7 & - & 35.4 & 139.6 & - & 6.6 \\
\hline 1812 & Dec & 8 & 15 & 34.4 & -117.7 & - & 6.9 \\
\hline 1812 & Dec & 21 & 19 & 34.2 & -119.9 & - & 7.1 \\
\hline- & - & - & - & - & - & - & - \\
\hline- & - & - & - & - & - & - & - \\
\hline- & - & - & - & - & - & - & - \\
\hline 1981 & Jan & 18 & $18: 17$ & 38.64 & 142.75 & 33 & 6.9 \\
\hline 1981 & Jan & 19 & $15: 11$ & -4.57 & 139.23 & 33 & 6.7 \\
\hline 1981 & Jan & 23 & $21: 13$ & 30.93 & 101.10 & 33 & 6.8 \\
\hline 1981 & Feb & 14 & $17: 27$ & 41.05 & 14.60 & 10 & 4.6 \\
\hline 1981 & Feb & 24 & $20: 53$ & 38.22 & 22.93 & 33 & 6.7 \\
\hline 1981 & Mar & 4 & $21: 58$ & 38.21 & 23.29 & 29 & 6.4 \\
\hline 1981 & Mar & 10 & $15: 16$ & 39.48 & 20.70 & 31 & 5.6 \\
\hline 1981 & Apr & 18 & $00: 32$ & -13.14 & -74.38 & 38 & 4.8 \\
\hline 1981 & Apr & 26 & $12: 09$ & 33.13 & -115.65 & 6 & 6.0 \\
\hline 1981 & May & 25 & $05: 25$ & -48.79 & 164.36 & 33 & 7.6 \\
\hline 1981 & Jun & 11 & $07: 24$ & 29.91 & 57.72 & 33 & 6.7 \\
\hline 1981 & Jun & 22 & $17: 53$ & -13.17 & -74.52 & 24 & 5.2 \\
\hline 1981 & Jul & 28 & $17: 22$ & 30.01 & 57.79 & 33 & 7.1 \\
\hline 1981 & Aug & 13 & $02: 58$ & 44.85 & 17.31 & 16 & 5.5 \\
\hline 1981 & Sep & 1 & 09:29 & -14.96 & -173.09 & 25 & 7.7 \\
\hline 1981 & Sep & 12 & $07: 15$ & 35.69 & 73.59 & 33 & 5.9 \\
\hline
\end{tabular}

These are typical recordings, and there is a general trend of more frequent earthquake recording at later times than earlier. This is probably due to technology improvement in addition to increasing human population and scientific interest. Unfortunately, there is no direct way to adjust this trend. However, this "pull of the recent" should not mask periodicity caused by the geophysical triggers suggested here. We made a time series based on the global earthquakes, by assigning ' 1 ' to the days with an earthquake $(M>4)$ and ' 0 ' to others. There are a few days of two earthquakes. These days were treated like single event days by assigning a ' 1 '. Excluding the events without date information, total number of event days is reduced to 3060. The initial section of this time series is illustrated in Figure 1a for events during two different time spans; 17 and 109 events for the first 2000 and 10000 days. Latitude dependence of the all earthquakes in the list is shown by ten degree intervals in Figure $1 \mathrm{~b}$. The concentration of observed events in the northern mid-latitudes may be in part affected by the dense seismic network there. The 
absence of earthquakes in the two polar regions may also be related to a lack of observational network, as well as simple areal considerations (there is more area between $0-10^{\circ}$ latitude than $80-90^{\circ}$ ).
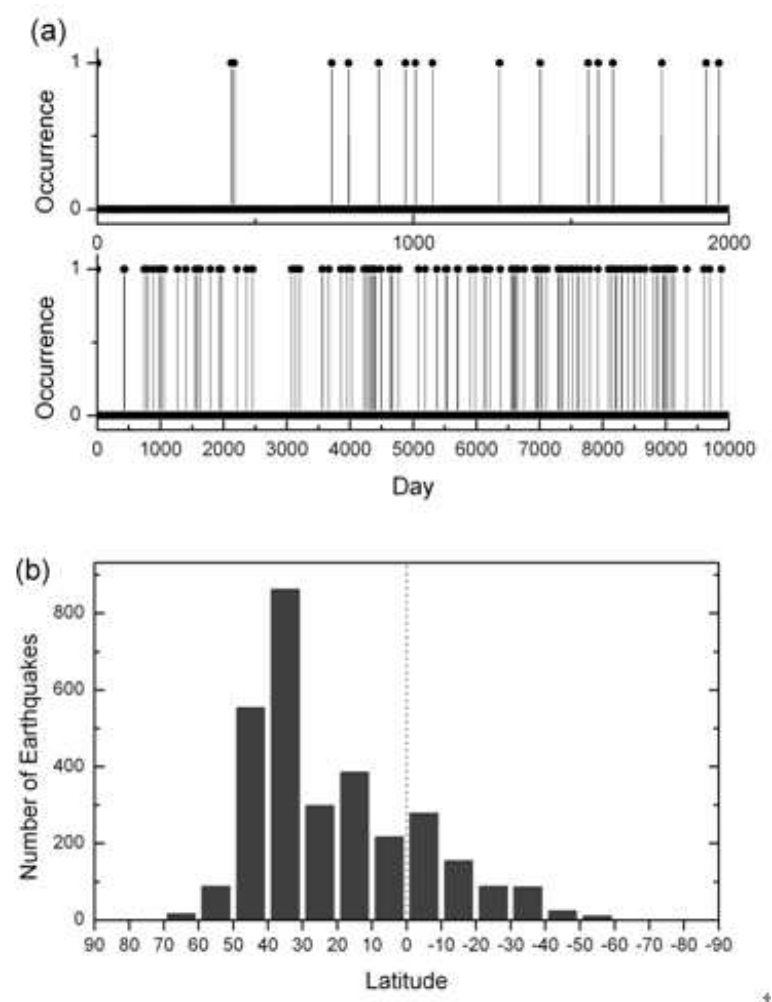

Figure 1. Time and spatial occurrence of global earthquakes of this study: (a) Global earthquake occurrence time series. 'O' refer to day of no earthquake, while ' 1 ' for day with earthquake $(M>4)$ regardless of the epicenter location. Events for the first 2000 days (top) and 10000 days (bottom). (b) Number of earthquakes that occurred in regions bounded by latitude parallels of 10 degree interval.

The Fourier power spectrum for the time series constructed for the global earthquake occurrence is shown in Figure 2. Since the time series is based on daily records, its Nyquist period corresponds to 2 days. However, about 18 earthquakes were recorded for each year on average, so periodicity shorter than a month cannot be distinguished. The total length of the time series was increased to about 718 years (262144 days) by zero-padding over five centuries in order to increase the resolution of the spectrum. Because the total data length is 195 years, periodicity comparable to or less than a century is meaningful. In Figure 2, the range of meaningful frequency is marked by vertical dotted lines.

There are many peaks that surpass then $95 \%$ significance level in the power spectrum for the time series of global earthquake occurrence (Fig. 2). At first glance, one might regard the acquired spectral peaks to represent random noise. To examine whether the peaks are related to geophysical phenomena, the spectrum is again shown at four selected period ranges in Figure 3. It is noted that true power should be evaluated by integration over the frequency domain. Two broad peaks at 42 and 29 years are noticeable in Figure 3a, but we know of no geophysical phenomena at those periodicities.

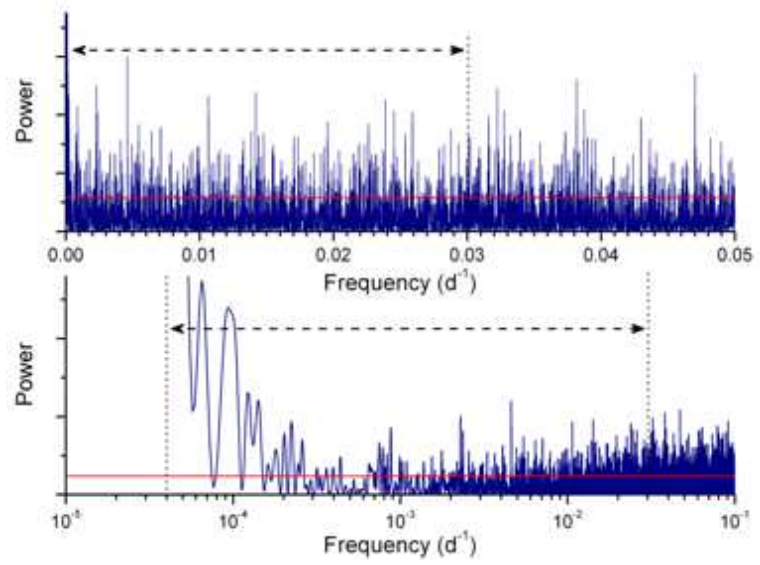

Figure 2. Fourier power spectra for the time series of global earthquake occurrence. Two spectra are in different horizontal scales. Reliable frequency range is marked by dotted arrows. Horizontal lines (in red) correspond to the $95 \%$ significant level.

Similarly, we cannot ascribe a specific geophysical mechanism to trigger the 6.3 and 3 4 year peaks in Figure $3 \mathrm{~b}$. Two other periodicities of 12.3 and 10.5 years might be associated with the 11 year solar irradiance cycle. Between 100 and 1000 days, Chandler and semi-Chandler periodicities are prominent. Although its amplitude is only a few meters, Chandler wobble brings periodic deformation in the Earth's crust and mantle so that earthquakes can be triggered by the wobble-induced deviatoric stress. The Chandler peak is split on the spectrum between 423 and 437 days (Fig. 3c). The splitting of 14 days could be caused by fortnightly lunar tide or atmospheric oscillating disturbance of period between 10 and 20 days.
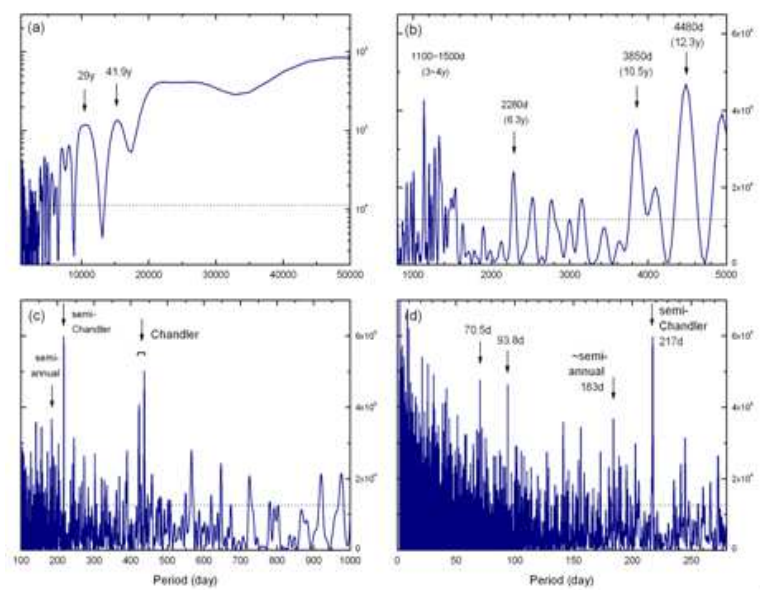

Figure 3. Fourier power spectra of global earthquake occurrence versus period. Each figures for four different period ranges are drawn together. Dotted lines are the 95\% significance levels. Each selected period bands are; (a) 1000-50000, (b) 800-5000, (c) 100-1000, and (d) 0-280 days.

While Fourier spectra faithfully yield amplitudes of sinusoidal components of given time series, the maximum entropy spectrum is known for its particular ability to 
reveal the signal periodicity, particularly when the time series is of limited duration and contaminated with large stochastic noise[15-16]. Figure 4 illustrates the spectrum for the time series of global earthquake occurrence acquired by the maximum entropy method. The spectrum is denoted as maximum likelihood (ML), because it is the harmonic mean of several maximum entropy spectra acquired for different filter lengths[17]. ML spectra of Figure 4 generally show the similar pattern with the Fourier spectra (Fig. 2) with reduced number of peaks.
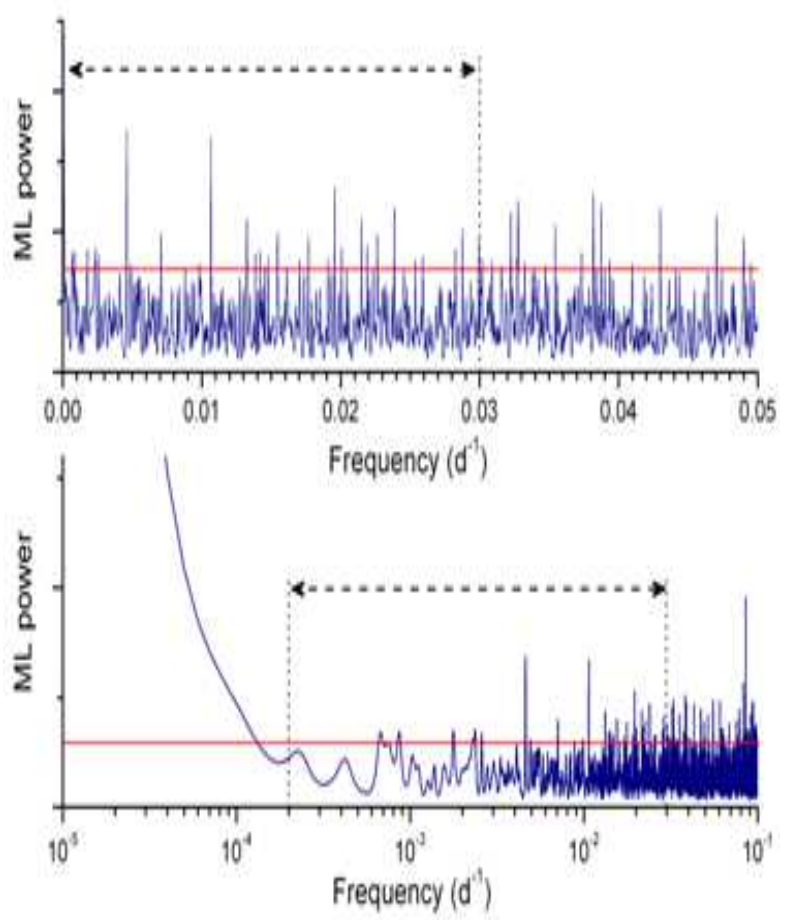

Figure 4. Maximum likelihood (ML) power spectrum for the time series of global earthquake occurrence. Reliable frequency range is marked by dotted arrows. Horizontal lines (in red) correspond to the $95 \%$ significance level.

To identify periodicity information directly, the same ML power spectrum is shown again as a function of period for two selected ranges; (a) 250 - 5000 and (b) 30 - 350 days (Fig. 5). Unlike the Fourier method, the maximum entropy method requires large memory storage proportional to the square of data length so that periodicity longer than 5000 days could not be practically estimated in the ML spectra of this study. Noticeable period peaks in the ML spectra are Chandler, semi-Chandler, and 94 days. Although their powers are not very large, these spectral peaks possibly show the effect on global earthquake triggering by the corresponding periodic phenomena, such as Chandler wobble. Other peaks are less obvious. The 76 day peak in the global earthquake occurrence spectrum might be associated with the periodicity of 75 - 80 days found from the atmospheric intra-seasonal oscillation in the mid latitude region[18]. The periodicity of 51 days might be affected by the same periodicity of solar irradiance[19].
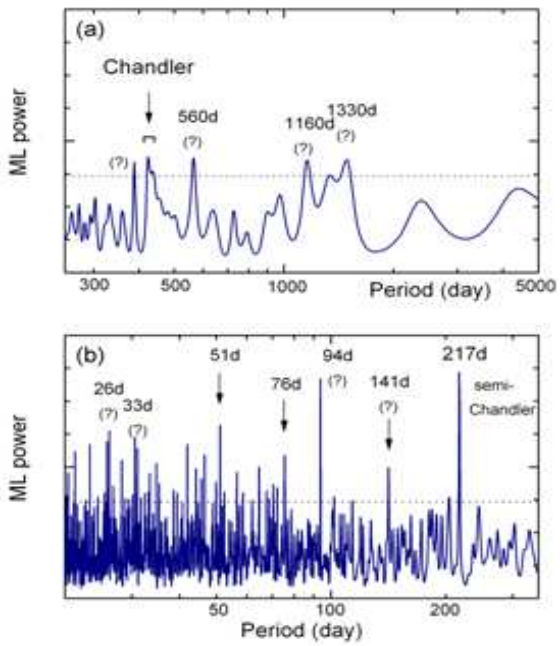

Figure 5. Maximum likelihood power spectra of global earthquake occurrence versus period. Figures for two period ranges are drawn together. Dotted lines are the 95\% significance levels.

\subsection{Historical Korean Earthquakes}

Korea is located in an intra-plate region of the Eurasian plate, several hundred kilometers from Japan trench - one of the most seismically active region in the world. Earthquake occurrence is much less frequent in Korea than it is in Japan. Nevertheless, more than a thousand earthquakes have been recorded in the Korean peninsula over the last millennium. Lee and Yang[20] compiled these earthquakes (Table 2) by referring to eight different kinds of old Korean governmental records, including 'Joseon Wangjo Sillok' (Annals of Choson Dynasty, 1392 - 1904 A.D.) and 'Goryeosa' (History of Goryeo, 1012 - 1392 A.D.).

http://sillok.history.go.kr/main/main.jsp, in Korean with English guide, last accessed in May 10, 2012.

http://osmstar.com.ne.kr/goreosajelo/kframe1.htm, in Korean with Chinese records, last accessed in May 10, 2012.

Table 2. Excerpts of Lee and Yang's historical Korean earthquakes catalog (2006). It is consisted of event number, date, location, and approximate magnitude estimate.

\begin{tabular}{rrcccc}
\hline Num & Year & Mon \& Day & Lat(N) & Long(E) & Mag \\
\hline 1 & 2 & Aug ?? & 41.3 & 125.4 & 3.5 \\
2 & 13 & May ?? & 37.4 & 127.3 & 3.5 \\
3 & 13 & Jun ?? & 37.4 & 127.3 & 3.5 \\
4 & 19 & Jan ?? & 41.2 & 126.2 & 3.5 \\
5 & 27 & Oct ?? & 37.4 & 127.3 & 6.4 \\
6 & 37 & Nov ?? & 37.4 & 127.3 & 3.5 \\
& 1012 & Apr 02 & 35.8 & 129.3 & 3.5 \\
96 & 1013 & Feb 03 & 35.8 & 129.3 & 3.5 \\
97 & 1013 & Apr 09 & 35.8 & 129.3 & 3.5 \\
98 & 1013 & Apr 28 & 35.3 & 128.5 & 3.5 \\
99 & 1013 & Dec 30 & 35.3 & 128.5 & 5.2 \\
100 & 1093 more earthquakes) & & \\
& 1904 & Jan 09 & 37.6 & 127.0 & 3.5 \\
2184 & 1904 & Mar 23 & 37.6 & 127.0 & 3.5 \\
\hline
\end{tabular}


Although the total number of earthquakes in Lee and Yang's catalog is 2185, only 95 earthquakes were recorded in the catalog during the first 1010 years. Clearly this is an incomplete record, rather than a systematic change in seismicity. Consequently, these initial spotty records are excluded from our analysis. Some records show interdependency potentially resulting from (i) duplicate reports from a single event felt in multiple locations, (ii) earthquake swarms, and (iii) main shock - aftershock relationships. Of these, we do not find any evidence for the third case. Following is one example of a suspected redundant listing - case (i);

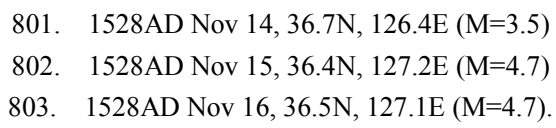

There are other examples showing earthquake-swarm behavior or redundant reporting between May 29, 1565 and May 19, 1566 at one location of 126.1E and 38.9N (near Pyeongyang). One earthquake was reported every day at the same place and of the same magnitude over the course of nearly a year. An excerpt of this record follows, suggesting possibility (ii);

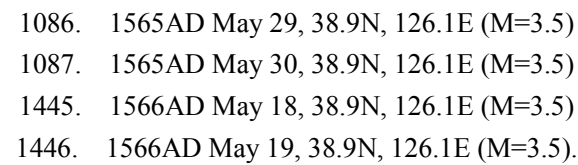

Since the instrumental observations also show the relatively dense seismicity in this region, this seismicity can possibly be considered a type of earthquake swarm.

In this study, we need to isolate the class of background seismicity, i.e. seismicity that is independent of all preceding seismicity, no matter what the possibilities are. To do this, the earthquake catalog was declustered by checking the event listing line by line. After these modifications of the dataset of Lee and Yang, another time series was constructed, now composed of the 1381 Korean earthquakes that occurred during 892 years between 1012AD and 1904AD. The initial part of this time series is illustrated in Figure 6 for two different time intervals; 8 events for the first 1000 days and 24 events for the first 10000 days.

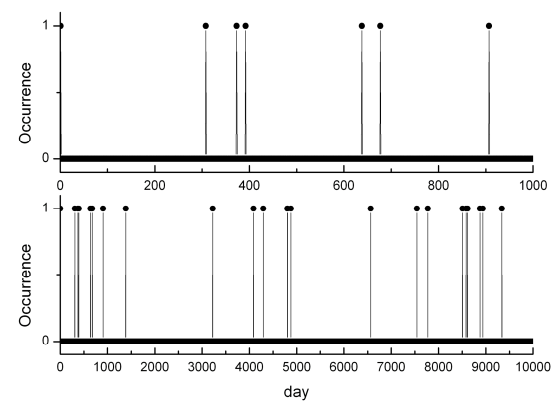

Figure 6. Historical Korean earthquake occurrence time series. ' 0 ' refers to days of no earthquake, while ' 1 ' for those days with any earthquakes $(M>3.5)$ in the Korean peninsula. Events for the first 1000 days (top) and 10000 days (bottom). Dataset acquired from Lee and Yang (2006) and further refined.
To reveal periodicity in the Korean earthquake occurrence, power spectra were calculated by the Fourier transform and maximum entropy method similarly as done for global earthquakes. Acquired spectra are shown versus frequency or period in Figure 7. Conspicuous one year (365day) periodicity dominates all other small peaks in both Fourier and ML spectra. In Figure 7c, two spectral peaks of 3500 and 2020 days (9.6 and 5.5 years) are marked with arrows. Although not shown in Figure 7, a periodicity of 38 years was found comparatively large and noticeable in the Fourier spectrum. These long period peaks are quite narrow on the spectrum versus frequency, i.e., having much smaller power than one year periodicity, and their geophysical drivers, if any, are not recognized.

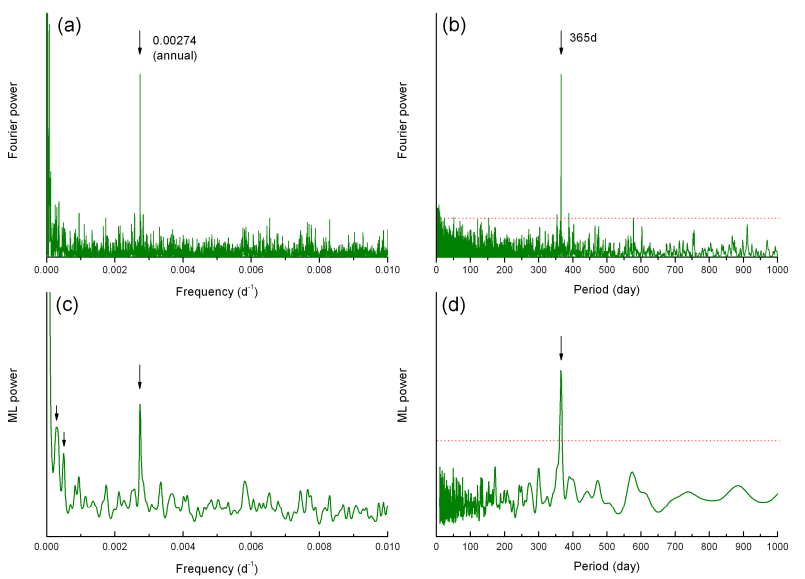

Figure 7. Power spectra acquired for the time series of historical Korean earthquakes: (a-b) Fourier power spectra versus frequency and period, (c-d) Maximum likelihood power spectra versus frequency and period. Two horizontal lines are the $99 \%$ significance level.

To determine whether the one year periodicity found in the spectrum is reflected as a seasonal concentration of earthquake occurrence, the cumulative number of Korean earthquakes occurring in each month is plotted in Figure 8.

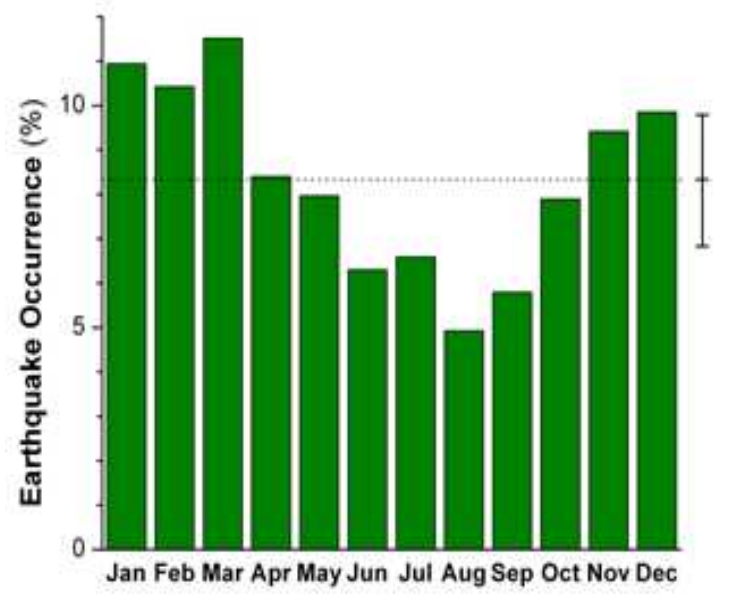

Figure 8. Monthly occurrence of the historical Korean earthquakes (for 1381 events in 893 years). The dotted line is annual average, and the two error bars correspond to deviations of $\pm 2 \sigma$. 
More Korean earthquakes have occurred during December to March, while less occurred in summer, particularly in August. It is noted here that more or less the same patterns in both the spectrum and monthly occurrence have been found from the unmodified dataset of Lee and Yang[20] - without removing suspected redundancy classified above as (i), (ii), and (iii). The raw data reveal a slightly smaller peak at one year periodicity and slightly diminished seasonal bias.

\subsection{Recent Korean Earthquakes}

Two datasets of recent Korean earthquakes were acquired from the Korea Meteorological Administration (KMA) and the Korea Institute of Geoscience and Mineral Resources (KIGAM) for this study. KMA keeps records of earthquakes that have occurred in and around Korean peninsula since 1978, and KIGAM does in its similar way since 1994. Seasonal concentration of recent Korean earthquake occurrence is plotted in Figure 9. The two datasets used for this study are distinct in their time spans and also in magnitude limits $(\mathrm{M}>2.0$ for KMA and $\mathrm{M}>$ 1.5 for KIGAM). The number of earthquakes in the two datasets are 965 (KMA) and 920 (KIGAM).

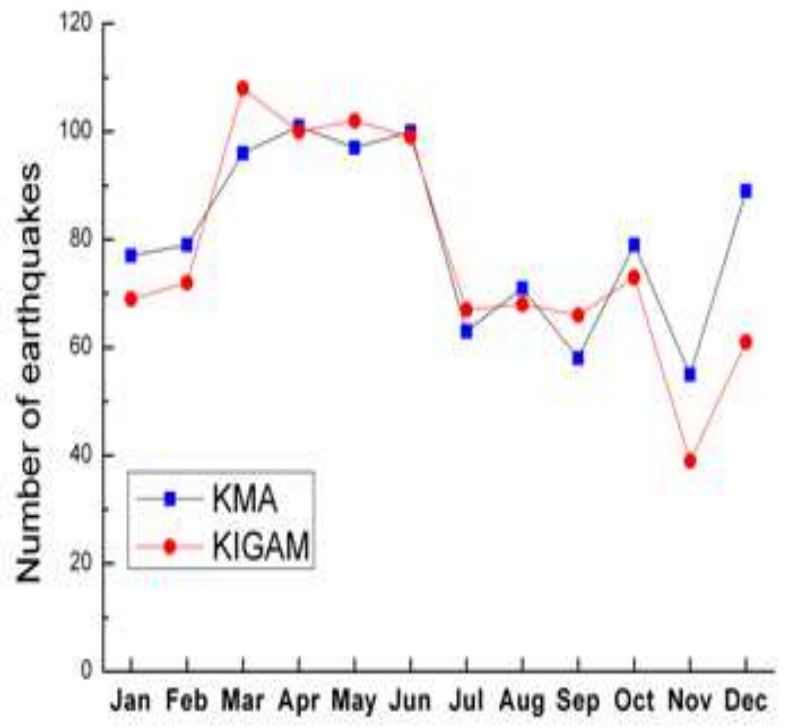

Figure 9. Cumulative occurrence numbers of recent Korean earthquakes for each month. Both KMA and KIGAM records are shown. Time span and magnitude limits are different for two records.

It is found that more Korean earthquakes occurred in the spring season between March to June, while less earthquakes occurred in the fall season between July and November. The epicenters of these earthquakes are plotted in Figure 10, where two seasonal earthquakes are distinctly shown (spring: Mar-Jun, fall: Jul-Nov). The epicenters are widely distributed over the Korean peninsula and two seasonal earthquakes seem to occur filling the regional gap except the small central area of the peninsula.
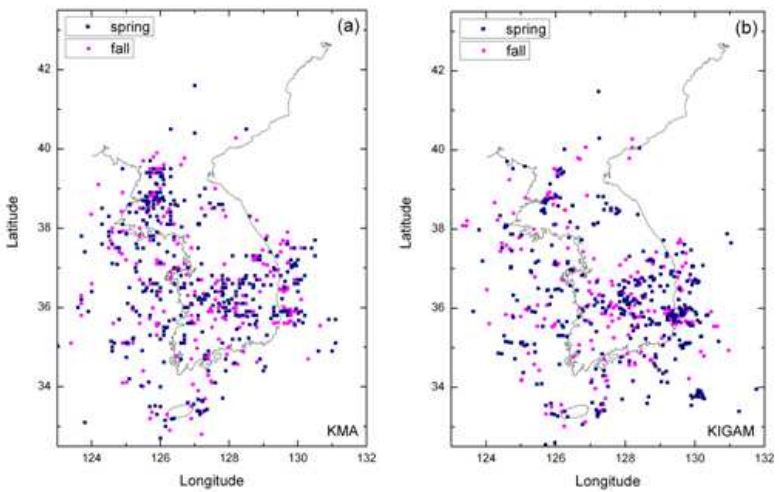

Figure 10. Epicenters of the recent Korean earthquakes. (a) KMA record, (b) KIGAM record. The earthquakes occurred in spring season (Mar-Jun) are indicated as blue squares, while those ones occurred in fall season (Jul-Nov) are shown pink circles.

\subsubsection{KMA Record}

For this study, a list of 965 Korean earthquakes (between 1978 and 2011: $M>2.0$ ) was taken from KMA record. A time series was constructed by the same way as others above, and its Fourier and maximum likelihood spectra were calculated (Fig. 11). One year periodicity exists in the all calculated spectra, but its dominance is not as conspicuous as in the longer record of historical Korean earthquakes. The overall character of these spectra is closer to white noise, and most peaks cannot be ascribed to any known geophysical origin.
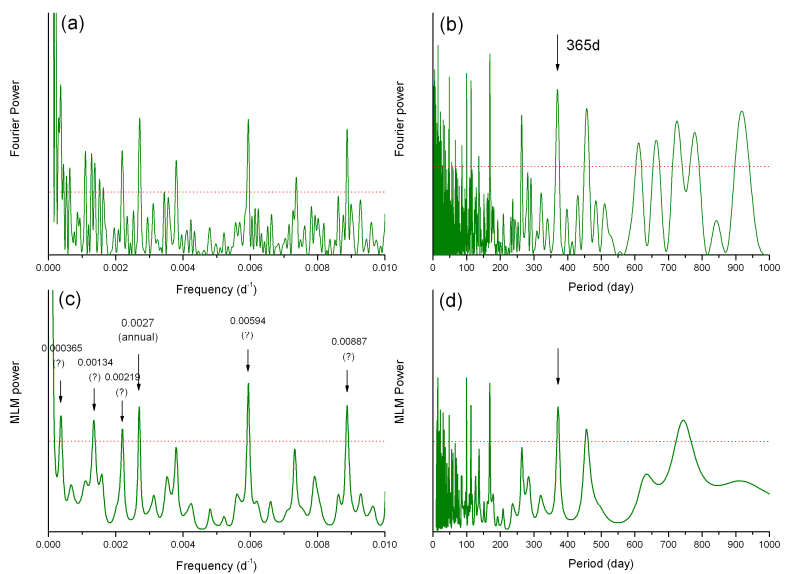

Figure 11. Power spectra acquired for the time series of recent Korean earthquakes (KMA record): ( $a-b)$ Fourier power spectra versus frequency and period, (c-d) Maximum likelihood power spectra versus frequency and period. Four horizontal lines correspond to the each $95 \%$ significance levels.

\subsubsection{KIGAM Record}

For a list of 920 Korean earthquakes (between 1994 and 2011: $M>1.5$ ) taken from KIGAM record, another time series was constructed similarly as before, and then its Fourier and maximum likelihood spectra were calculated (Fig. 12). The general character of these spectra are again closer to white noise than those of historical Korean earthquake. The spectra again contain numerous peaks that 
cannot be ascribed to any particular geophysical origin. For example, a conspicuous 1110-day periodicity can be seen in all the spectra. However, one year periodicity appears stronger than in the spectrum for the KMA record, and fortnightly periodicity peak exists over the $95 \%$ significance level. Though not shown in the frequency spectra, peaks of monthly periodicity seem to exist in the two period spectra.
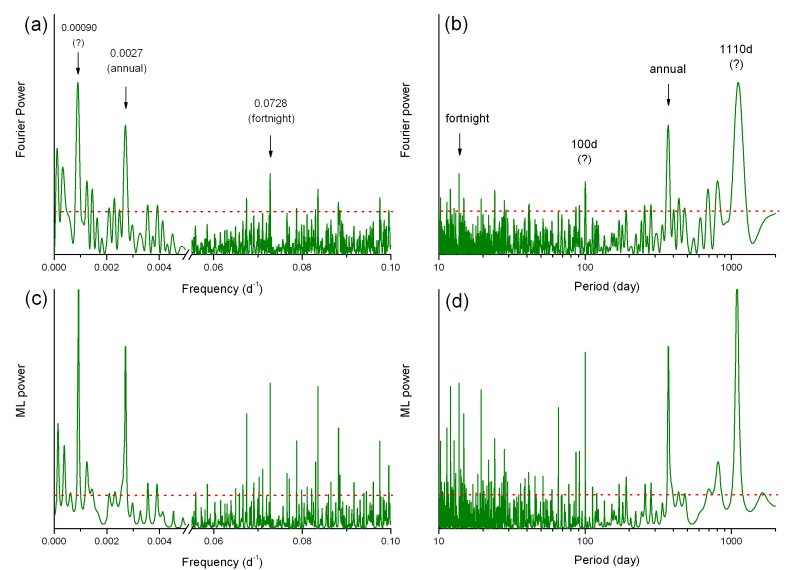

Figure 12. Power spectra acquired for the time series of recent Korean earthquakes (KIGAM record): (a-b) Fourier power spectra versus frequency and period, (c-d) Maximum likelihood power spectra versus frequency and period. Red horizontal lines are the $95 \%$ significance levels. For convenience, breaks are made in the two frequency axes.

\subsection{GPS Displacement Measured at DAEJ}

Plate tectonic movement was confirmed to occur rather uniformly over the southern part of the Korean peninsula[21]. Located at the center of South Korea, the city of Daejeon is an ideal place to monitor the displacement of the Korean crust as a whole. DAEJ (Daejeon) is one of the IGS permanent GPS sites, and has been stably operating since 1999. In Figure 13, GPS derived displacement of DAEJ is illustrated after excluding a linear trend due to plate tectonic movement. The components of annual and semi-annual oscillations determined as the least square error fit, are shown together with the data in Figure 13a. Annual vertical oscillation is the largest periodic component at about $6 \mathrm{~mm}$ in amplitude, and eastward and northward oscillations are within $\pm 3 \mathrm{~mm}$. From the vertical oscillation fit curve, the maximum annual depression of DAEJ is found to occur in December (around Dec $\left.10^{\text {th }}\right)$. The data and fit curves are illustrated again in finer amplitude scale for shorter time span between 2009 and 2011 in Figure 13b. There are marked rectangular boxes, which correspond to portions of curves having large ratio of tangential stress versus normal stress associated with each directional oscillation (for related phenomena, see[9]). Therefore, it is speculated that earthquakes would preferentially occur during those times.
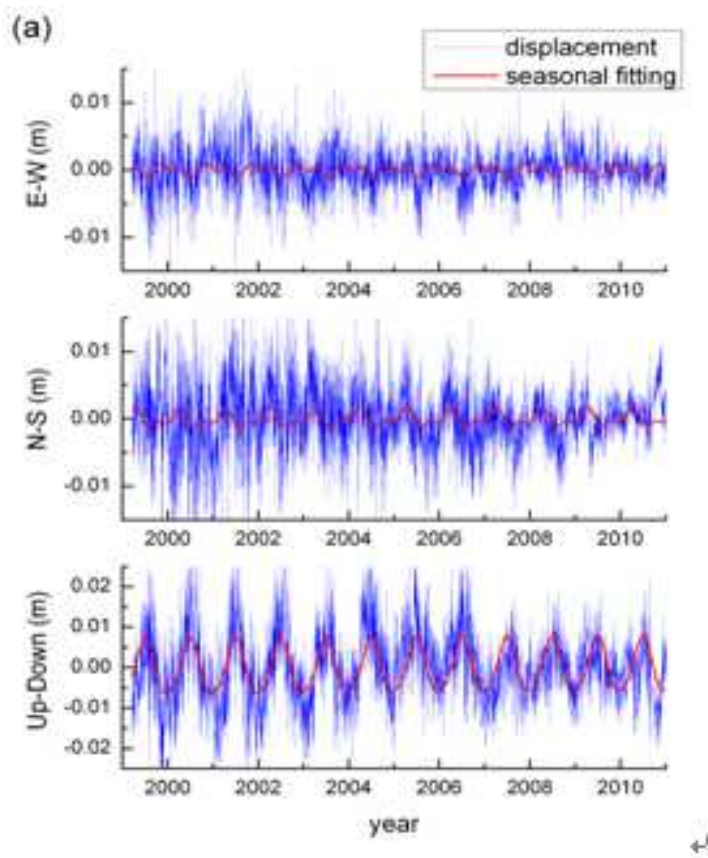

(b)
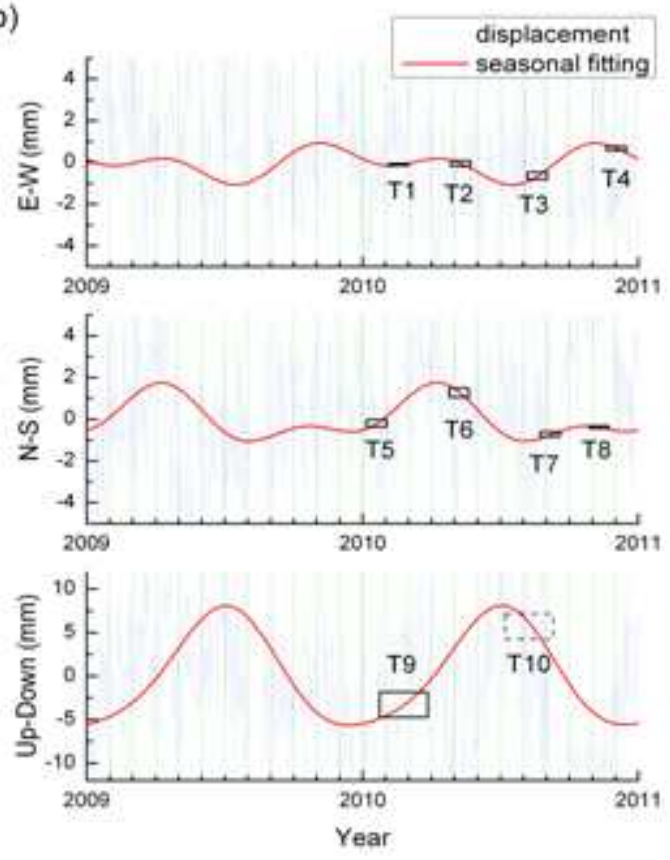

Figure 13. Three components of displacement of DAEJ GPS site (Daejeon, Korea) monitored between 1999 and 2011: (a) Datasets are illustrated with least square fit curves for annual and semiannual oscillation for total time span, (b) Same fit curves are drawn in finer vertical scale and shorter time span 2009 - 2011. Rectangular boxes correspond to phases of maximum ratio of tangential stress versus normal stress.

The three oscillating displacement signals (name each as $y(t))$ are not purely sinusoidal of single frequency, so that Cochran's[9] choice of 45 degree phase delay for rupture was not exactly taken here. Each marking box was drawn following Cochran's[9] criteria as follows: Times of the maximum tangential/normal stress ratio, i.e. Tk (T1, T2, . . T10), were determined as middle of $t_{i}$ and $t_{j}$, where $y^{\prime}\left(t_{i}\right)=0$ and $y^{\prime \prime}\left(t_{j}\right)=0$ respectively $\left(t_{j}>t_{i} ; t_{j}\right.$ follows after $t_{i}$ ). For the two perpendicular horizontal 
oscillations (E-W and N-S), each set of four particular times were determined. However, for the vertical oscillation, two times only (T9 and T10) were determined (due to the dominance of annual signal). These small oscillations lead to additional minute stresses. However, unlike horizontal oscillation, additional stress due to vertical displacement leads to only one effective triggering time in one period (during the intermediate state changing from additional compression to relaxation), so that $\mathrm{T} 9$ is relevant while T10 is not. From the East-West displacement, August to September (T3) and late November to early December (T4) are the more likely season of earthquake. Similarly, late April to May (T6) and January (T5) are those associated with North-South oscillation. Each height of the marking boxes are proportional to the range, which is the difference between adjacent $y_{\max }$ and $y_{\min }$, i.e., $\left|y_{\text {extreme }}\left(t_{n}\right)-y_{\text {extreme }}\left(t_{n+1}\right)\right|$. The box widths were chosen to be proportional to the each period involved, such as one month for semi-annual and two months for annual oscillations.

\section{Discussion}

The Chandler periodicity found in global earthquake occurrence manifests the effect of the periodic perturbation induced in the Earth's crust due to the Chandler wobble. The pole offset from the Earth's principal $z$-axis is usually a few meters. It is important that the $z$-axis here is meant to be the principal axis not the Reference Pole (formerly called the Conventional International Origin), although polar motion is usually referenced to the Reference Pole[22]. The pole offset induced deformation in the Earth's crust is small (in the order of one centimeter), but exists over the globe[23]. Because this deformation is of period longer than the fault nucleation time, it can contribute to earthquake triggering[24]. Conversely, short period additional stress oscillations due to tides do not result in clear indication of earthquake triggering effect notwithstanding their large amplitude[24]. A periodicity of 13-16 months in the slow slip events occurred at the circum-Pacific subduction zones extended over $10000 \mathrm{~km}$, which was possibly related to deformation induced by Chandler wobble[14].

The strong annual periodicity in the spectra of historical Korean earthquakes is related to the large spring-fall seasonal contrast (Fig. 8). There are diverse geophysical phenomena showing seasonal perturbation. Three strong candidates for the primary annual perturbation responsible for triggering historical Korean earthquake are follows; (i) local seasonal surface loading by atmospheric pressure and precipitation, (ii) regional tectonic oscillation caused by global deformation mode as suggested by Blewitt et al. (2001), and (iii) seasonal variation of ground water storage in the crust. Each of these different phenomena could introduce comparable contributions. However, seasonal atmospheric/hydrologic loading (i.e., (i) and (iii)) is believed to be the main cause of annual vertical oscillation of the Korean peninsula as a whole. Horizontal oscillation should come from the tectonic oscillation.

The annual periodicity is also confirmed in the recent Korean earthquakes (Fig. 11-12). However, there is a shift of about two months delay in the seasonal concentration of the recent Korean earthquakes compared with historical ones, and the cause for which is unknown. A systematic error of order of ten days in lunar to solar calendar date conversion for the historical Korean earthquake data is suspected as a partial explanation[26]. Existence of fortnight periodicity in the KIGAM data may show the triggering effect of lunar tide or its associated phenomena on Korean earthquakes.

The twelve year monitoring at Daejeon, Korea (Fig. 13a) clearly shows annual and semiannual oscillation of that site. According to the maximum tangential/compressional stress ratio criterion, seasons of greater earthquake likelihood are evident. It is noted that the area of the boxes drawn in Figure $13 \mathrm{~b}$ can approximately represent the relative effect of triggering, because they are proportional to 'amplitude $\times$ oscillation period'. A greater triggering effect would thus be expected in late January to early March associated with the vertical seasonal oscillation. Overall annual vertical oscillation of Korean crust is thought to be dominated by local seasonal surface loading by atmospheric pressure and precipitation, while the seasonal horizontal oscillation might be strongly affected by regional motion extended over more than a thousand kilometers. By considering the earthquake triggering effect due to the seasonal oscillation of Korean crust inferred from GPS displacement, concentration of Korean earthquakes in the season from February to April can be expected. These interpretations generally agree with the seasonal concentrations of the Korean earthquake occurrences.

A few further remarks are given below. The NOAA global earthquake record has more frequent event listings for later years (of total 200 (195) year data), and we did not attempt to adjust for this tendency. Regarding any concerns about errors introduced by this observational "pull of the recent," the trend would be expected to add peaks of Nyquist frequency and its multiple harmonics, such as 100 , $50,33,25,20 \ldots$ years, all of which are much longer than the main period range of interest. Therefore we do not expect that a better early observational record would alter the results indicated in Figures 2 - 5.

The comparatively much weaker annual signal in the global earthquake occurrence spectrum can be attributed to locally different and largely varying phase in seasonal variations in the northern and southern hemispheres. This absence possibly implies that the Blewitt's[25] global deformation mode tends to be screened by locally different phase of seasonal variation. However, existence of 300 or 76 day periodicities suggests that the spatial influence of the geophysical processes responsible for those two periodicities extend to thousands of kilometers.

Non-existence of Chandler periodicity in the Korean earthquake occurrence can be explained by the fact that 
Korean peninsula is a small land area of dimension of about $300 \mathrm{~km} \times 1000 \mathrm{~km}$. In order for the earthquake triggering effect associated with pole offset to be visible in a seismic record, the area involved must be large enough to involve the in-phase global oscillatory deformation as a whole.

\section{Conclusion}

In this study, four different kinds of data were analyzed; (1) time series based on NOAA global earthquake occurrence record for last two centuries, (2) time series based on the historical Korean earthquake occurrence record for about nine centuries, (3) recent instrumental Korean earthquake record for recent decades, and (4) twelve years of GPS displacement at Daejeon nearly located at the center of South Korea. For each time series, spectral analysis was conducted to reveal any differences from completely random noise. Among the resulting peaks above $95 \%$ significance level on the global earthquake spectrum, the most meaningful ones are the Chandler and semi-Chandler periodicities. Existence of these two is ascribed to the earthquake triggering effect of Chandler wobble. For the historical Korean earthquake time series, a strong one year periodicity emerged through spectral analysis, and this is interpreted to be caused by the seasonal oscillation of the Korean crust, further confirmed by the twelve year GPS monitoring at Daejeon. The amplitude of horizontal oscillation of Korean crust is about one third of the vertical oscillation. The annual oscillation of the Korean crust is mainly ascribed to the winter to summer atmospheric/hydrologic loading cycle. Recent Korean earthquake occurrence also displays a seasonal concentration similar to the long-term historical data.

\section{Acknowledgments}

This study was supported by one of the Basic Core Technology Development Programs of Korea Astronomy and Space Science Institute (KASI). The authors thank NOAA and USGS for supporting useful dataset of global earthquake occurrence, and they also thank KMA and KIGAM for the recent Korean earthquake records. They also appreciate Drs. Lee, K. and Yang, W. S. for their list of historical Korean earthquakes. Finally they thank Dr. Baek, J. (deceased) for his work to infer DAEJ displacement from raw GPS data.

\section{References}

[1] B. Gutenberg, C. F. Richter, "Frequency of Earthquakes in California," Bulletin of Seismological Society of America, vol. 34, pp. 185-188, 1944.

[2] S. C. Han, C. K. Shum, C. J. Bevis, C. Y. Kuo, "Crustal Dilatation Observed by GRACE after the 2004 Sumatra-Andaman Earthquake," Science, vol. 313, pp. 658-662, 2006.
[3] S. K. Park, W. Dalrymple, J. C. Larsen, "The 2004 Parkfield earthquake: Test of the electromagnetic precursor hypothesis," Journal of Geophysical Research, vol. 112, B05302, 2007.

[4] M. C. Lee, R. Pradipta, W. J. Burke, A. Labno, L. M. Burton, J. A. Cohen, S. E. Dorfman, A. J. Coster, M. P. Sulzer, S. P. Kuo, "Did Tsunami-Launched Gravity Wave Trigger Ionospheric Turbulence over Arecibo?," Journal of Geophysical Research, vol. 113, A01302, 2008.

[5] Y. Bock, D. Melgar, B. W. Crowell, "Real-Time Strong-Motion Broadband Displacements from Collocated GPS and Accelerometers," Bulletin of Seismological Society of America, vol. 101, pp. 12904-2925, 2011.

[6] S. Ozawa, T. Nishimura, H. Suito, T. Kobayashi, M. Tobita, T, Imakiire, "Coseismic and postseismic slip of the 2011 magnitude-9 Tohoku-Oki earthquake," Nature, vol. 475, pp. 373-376, 2011.

[7] K. Heki, "A Tale of Two Earthquakes," Science, vol. 332, pp.1390-1391, 2011.

[8] L. Métivier, O. de Viron, C. P. Conrad, S. Renault, M. Diament, G. Patau, "Evidence of earthquake triggering by the solid earth tides," Earth and Planetary Science Letters, vol. 278, pp. 370-375, 2009.

[9] E. S. Cochran, J. E. Vidale, S. Tanaka S., "Earth Tides Can Trigger Shallow Thrust Fault Earthquakes," Science, vol. 306, pp. 1164-1166, 2004.

[10] M. Tolstoy, F. L. Vernon, J. A. Orcutt, F. K. Wyatt, "Breathing of the seafloor: Tidal correlations of seismicity as Axial volcano," Geology, vol. 30, pp. 503-506, 2002.

[11] S. Tanaka, M. Ohtake, H. Sato, "Evidence for tidal triggering of earthquakes as revealed from statistical analysis of global data," Journal of Geophysical Research, vol. 107, B10, 2211, 2002.

[12] T. H. Heaton, "Tidal Triggering of Earthquakes," Bulletin of Seismological Society of America, vol. 72, pp. 2181-2200, 1982.

[13] J. E. Vidale, D. C. Agnew, M. J. S. Johnston, D. H. Oppenheimer, "Absence of earthquake correlation with Earth tides: An indication of high preseismic fault stress rate," Journal of Geophysical Research, vol. 103, B10, pp. 24567-24572, 1998.

[14] Z. K. Shen, Q. Wang, R. Bürgman, Y. Wan, J. Ning, "Pole-Tide Modulation of Slow Slip Events at Circum-Pacific Subduction Zones," Bulletin of the Seismological Society of America, vol. 95, pp. 2009-2015, 2005.

[15] T. J. Ulrych, "Maximum Entropy Power Spectrum of Truncated Sinusoids," Journal of Geophysical Research, vol. 77, pp. 1396-1400, 1972.

[16] R. T. Lacoss, "Data Adaptive Spectral Analysis Method," Geophysics, vol. 36, pp. 661-675, 1971.

[17] J. P. Burg, "The Relationship between Maximum Entropy Spectra and Maximum Likelihood Spectra," Geophysics, vol. 37, pp. 375-376, 1972.

[18] D. Pancheva, N. J. Mitchell, P. T. Younger, H. G. Muller, H. $\mathrm{G}$., "Intra-seasonal oscillation observed in the MLT region 
above UK $\left(52^{\circ} \mathrm{N}, 2^{\circ} \mathrm{W}\right)$ and ESRANGE $\left(68^{\circ} \mathrm{N}, 21^{\circ} \mathrm{E}\right)$, Geophysical Research Letter, vol. 30, 2084, 2003.

[19] S. D. Bouwer, "Periodicities of Solar Irradiance and Solar Activity Indices, II," Solar Physics, vol. 142, pp. 365-389, 1992.

[20] K. Lee, W. Yang, "Historical Seismicity of Korea," Bulletin of Seismological Society of America, vol. 96, pp. 846-855, 2006.

[21] S. Jin, P. H. Park, "Strain accumulation in South Korea inferred from GPS measurements," Earth Planets Space, vol. 58, pp. 529-534, 2006.

[22] R. S. Gross, "Earth Rotation Variation - Long period", in
GEODESY - Treatise on Geophysics vol. 3 ed. by T. Herring, Elsevier, Amsterdam, 2009.

[23] J. M. Wahr, "Deformation Induced by Polar Motion," Journal of Geophysical Research, vol. 90, B11, pp. 9363-9368, 1985.

[24] N. M. Beeler, D. A. Lockner, "Why earthquakes correlates weakly with the solid Earth tides: Effects of periodic stress on the rate and probability of earthquake occurrence," Journal of Geophysical Research, vol. 108, B8, 2391, 2003.

[25] G. Blewitt, D. Lavallée, P. Clarke, K. Nurutdinov, "A New Global Mode of Earth Deformation: Seasonal Cycle Detected," Science, vol. 294, pp. 2342-2345, 2001.

[26] K. Lee, personal communication, 2012. 